\title{
Drug-eluting stents in the management of peripheral arterial disease
}

\author{
Marc Bosiers' \\ Catherine Cagiannos' \\ Koen Deloose' \\ Jürgen Verbist ${ }^{2}$ \\ Patrick Peeters ${ }^{2}$ \\ 'Department of Vascular Surgery, \\ AZ St-Blasius, Dendermonde, Belgium; \\ ${ }^{2}$ Department of Cardiovascular and \\ Thoracic Surgery, Imelda Hospital, \\ Bonheiden, Belgium
}

\begin{abstract}
Since major meta-analyses of randomized controlled trials in interventional cardiology showed the potential of drug-eluting stents in decreasing restenosis and reintervention rates after coronary artery stenting, one of the next steps in the treatment of arterial occlusive disease is the transfer of the active coating technology towards peripheral arterial interventions. In this manuscript, we aim to provide a literature overview on available peripheral (lower limb, renal, and supra-aortic) drug-eluting stent applications, debate the cost implications, and give recommendations for future treatment strategies.

Keywords: critical limb ischemia, drug-eluting stent, below the knee, infrapopliteal, crural, limb salvage
\end{abstract}

Endovascular interventional techniques have been used to treat peripheral arterial occlusive disease (PAOD) since their conception and, despite many improvements, they remain the subject of continued modifications. Although percutaneous transluminal angioplasty (PTA) and stenting are now widely accepted and are even gradually replacing traditional open peripheral vascular surgical approaches, there remains one significant limiting factor: post-angioplasty and in-stent restenosis. Major meta-analyses of randomized controlled trials (RCT) from interventional cardiology show that in coronary artery disease, the use of drug-eluting stents (DES) has resulted in decreased restenosis and reintervention rates compared to bare metal stents (BMS) (Hill et al 2004; Mauri et al 2005; Roiron et al 2006). There is heterogeneity between outcomes depending on different drug coatings (sirolimus and its derivates versus paclitaxel and its derivates), suggesting higher efficacy with sirolimus-eluting stents. This manuscript reviews the literature for the available applications of DES in the management of PAOD and presents recommendations for future treatment strategies.

\section{Methods}

We reviewed publications listed in MEDLINE that presented applications of DES in noncoronary arterial disease from 1950 up to March 2007. The key word searched was "stent" combined with terms describing stent coating, "eluting, paclitaxel, sirolimus", and lesion location, "peripheral, infrapopliteal, below-the-knee, crural, femoral, iliac, renal, carotid, intravascular, extravascular". In addition, relevant abstracts and presentations from pertinent conferences (TCT, CIRSE, Charing Cross and Euro-PCR) were identified and the reference sections of retrieved articles were manually screened. Furthermore, ongoing unpublished trials were identified by accessing www.clinicaltrials.gov.

\section{Results}

Drug-eluting stents have been used to ameliorate outcomes in the treatment of atherosclerotic occlusive disease in various noncoronary locations. We believe that DES 
may influence outcomes depending on the vascular bed treated, thus, below are presented results subdivided into anatomic regions.

\section{Lower limb arteries Femoropopliteal vasculature}

The investigators of the SIROCCO trial (SIROlimus Coated COrdis Self-expandable Stent), were the first to publish the outcomes following use of sirolimus-eluting (SES) stents in the infrainguinal vasculature. This double-blind RCT evaluated the efficacy of sirolimus-eluting self-expanding nitinol stents versus the same commercially available bare metal stents (SMART ${ }^{\circledR}$, Cordis. Miami, FL, USA) in the superficial femoral artery (SFA) (Duda et al 2002, 2005, 2006). The study was performed in 2 phases. After interim analysis of SIROCCO I revealed a high fracture rate, it was decided to reduce the maximal allowed lesion length from $20 \mathrm{~cm}$ to $14.5 \mathrm{~cm}$ in order to decrease the number of stents implanted from 3 to 2 in an attempt to avoid stent fractures (Duda et al 2002). In total, 93 patients with occlusions or stenoses (average lesion length $8.3 \mathrm{~cm}$ ) at the level of the SFA were included. The sirolimus-eluting SMART $^{\circledR}$ stent was implanted in 47 and the bare SMART ${ }^{\circledR}$ nitinol stent in 46 patients. (Duda et al 2006) Both groups were followed for a mean of 24 months and no significant differences were observed in either the peak velocity ratios (PVR), nor ankle-brachial index (ABI). At 24 months, the in-stent restenosis rate as measured by duplex ultrasound was not significantly different between the sirolimus and the bare metal stent group, being $22.9 \%$ versus $21.1 \%$, respectively. The target lesion revascularization (TLR) was $6 \%$ in the sirolimus group and $13 \%$ in the bare stent group. Higher values were found for target vessel revascularization (TVR): $13 \%$ and 22\%, respectively. (Duda et al 2006) In both groups at 24 months, no amputations were performed as a result of complications from stenting. The SIROCCO-investigators concluded that the study provided objective evidence supporting the safety and efficacy of drugeluting and bare metal self-expanding nitinol SMART ${ }^{\circledR}$ stents in patients with chronic limb ischemia (CLI) and TASC C SFA lesions. They stated that no significant difference could be found between the bare and the sirolimus-eluting SMART ${ }^{\circledR}$ stents, because the restenosis rate in the bare stent group was unexpectedly low (Duda et al 2002, 2005, 2006).

To date, SIROCCO is the only completed RCT that has published results showing the utility of DES in the femoropopliteal (FP) vasculature. At the preparation of this manuscript, 2 further clinical trials investigating the efficacy of different types of DES for the treatment of FP atherosclerotic occlusive disease are in the process of completing patient enrollment. It is hoped that publication of these ongoing trials will clarify whether DES are in fact superior to bare metal stents (BMS) in the FP area.

The first trial from Abbott Vascular Inc (Santa Clara, CA, USA) has completed enrollment of 100 patients. Known as the STRIDES trial (SFA Treatment with Drug-Eluting Stents Study), this is a European prospective non-randomized controlled trial that aims to evaluate the safety and performance of the DYNALINK-E ${ }^{\circledR}$ Everolimus-Eluting Peripheral Stent System in above-knee (AK) FP de novo or restenotic lesions. The maximal length of lesion treated is $17 \mathrm{~cm}$. The second trial, the ZILVER ${ }^{\circledR} \mathrm{PTX}^{\mathrm{TM}}$ paclitaxel-eluting stent study from Cook Inc (Bloomington, IN, USA) is also completing enrollment. In this study, the safety and efficacy of the ZILVER ${ }^{\circledR}$ $\mathrm{PTX}^{\mathrm{TM}}$ stent is being evaluated in the AK FP vasculature for lesions up to $28 \mathrm{~cm}$ in length. This study has 2 components, the first being a randomized investigational device exemption (IDE) trial that compares 240 ZILVER $^{\circledR}{ }^{P T X}{ }^{\mathrm{TM}}$ patients with 240 control patients. The control patients undergo percutaneous transluminal angioplasty (PTA) followed by placement of ZILVER ${ }^{\circledR}$ BMS for procedural failures. The second phase is a Global Registry evaluating an extra 760 ZILVER $^{\circledR}$ PTX $^{\mathrm{TM}}$, thus, giving a total of 1000 patients who will have received the paclitaxel-coated ZILVER ${ }^{\circledR}$ stents for FP lesions. At the 2007 International Symposium on Endovascular Therapies (ISET) meeting in Miami (http://www.iset.org/), Michael Dake presented an interim report for the IDE-phase, on behalf of the ZILVER ${ }^{\circledR}$ PTX ${ }^{\mathrm{TM}}$ investigators. Data on the first 60 patients enrolled in the trial were presented. Twenty-eight patients were assigned to the ZILVER ${ }^{\circledR} \mathrm{PTX}^{\mathrm{TM}}$ group and 32 to the PTA control group. The mean follow-up duration was 9 months (range 6-18 months). Based on this preliminary data set, Dake concluded that the use of DES in AK FP lesions did not result in any safety concerns. The event-free survival was $89 \%$ in the ZILVER $^{\circledR}$ PTX $^{\mathrm{TM}}$ versus $91 \%$ in the PTA groups. Stent safety at 6 months was evaluated by plain X-ray, which showed no stent fractures in any of the implanted stents, and IVUS which did not reveal aneurysm formation or strut malapposition. Taking into account acute treatment failure, the investigators found a higher 9-month primary patency rate in the patients assigned to the ZILVER ${ }^{\circledR}$ PTX $^{\mathrm{TM}}$ treatment $(90 \%)$ in comparison to the PTA control group (52\%). Stent effectiveness as evaluated by preliminary results in this analysis leads us to anticipate an equally encouraging final trial outcome.

These 2 landmark DES trials are of utmost importance for the endovascular community because positive results could 
facilitate implementation of broad changes in the treatment paradigm of PAOD.

\section{Infrapopliteal vasculature}

The diameter similarities between infrapopliteal and coronary vessels make the use of DES in the BK vascular bed an intuitive application. Feiring and colleagues (2004) were the first to demonstrate the safety and utility of using coronary DES in the tibial vessels and paved the way for a more widespread application of DES for treating infrapopliteal disease.

Based on the outcome of 4 independent investigatorintiated studies, the sirolimus-eluting Cypher ${ }^{\circledR}$ stent (Cordis, Miami, FL, USA) have been CE-marked for the BK indication (Siablis et al 2005; Scheinert et al 2005; Bosiers et al 2006; Commeau et al 2006). Our own study (Bosiers et al 2006) evaluated the Cypher ${ }^{\circledR}$ stent for BK applications in 18 CLI patients (Rutherford 4 and 5) resulted in a 6-month limb salvage rate of $94.4 \%$ with an angiographic late lumen loss of only $0.38 \mathrm{~mm}$ in the surviving patients. Commeau and colleagues (2006) used SES to treat 30 consecutive patients with CLI (Rutherford category 3-6) and a minimum of 2 diseased infrapopliteal vessels. A limb salvage rate of 100\% was achieved at a mean follow-up of 7.7 months, and all surviving patients treated with Cypher ${ }^{\circledR}$ stents had marked clinical improvement with $97 \%$ primary patency as measured by target lesion revascularization (Commeau et al 2006). Siablis and colleagues (2005) compared the outcome of 29 CLI patients treated with the sirolimus-eluting Cypher ${ }^{\circledR}$ stent with another 29 CLI patients receiving a BMS for infrapopliteal revascularization. The 6-month primary patency rate was significantly higher in the Cypher ${ }^{(B)}$ group compared to the BMS group $(92.0 \%$ and $68.1 \%$, respectively, $\mathrm{p}<0.002)$ (Siablis et al 2005). Angiographic follow-up 6-months post index intervention revealed a binary in-stent restenosis of only $4.0 \%$ after Cypher ${ }^{\circledR}$ stent implantation, while the rate was as high as 55.3\% after BMS implantion ( $p<0.001)$ (Siablis et al 2005). Siablis and colleagues (2007) also published their one-year follow-up results showing $86.4 \%$ primary patency in patients with DES, whereas patients with BMS had primary patency of $40.5 \%$ ( $\mathrm{p}<0.001)$. Likewise, the binary in-stent restenosis was better with DES, 36.7\% in the Cypher ${ }^{\circledR}$ stent group, and $78.6 \%$ in the BMS group ( $p<0.001)$. (Siablis et al 2007). The same highly significant difference in 6-month angiographic outcome between the Cypher $^{\circledR}$ and the nondrug-eluting BX Sonic ${ }^{\circledR}$ stent (Cordis, Miami, FL, USA) was confirmed by Scheinert and colleagues (2006) in a nonrandomized controlled trial. The study evaluated 60 consecutive patients presenting with infrapopliteal lesions. The binary restenosis rate was found to be $0 \%$ in the Cypher $^{\circledR}$ arm and $56.5 \%$ in the control BMS arm $(\mathrm{p}<0.001)$ (Scheinert et al 2006).

Feiring and Wesolowski (2007) recently proposed a new technique for combining antegrade popliteal arterial access with tibial DES implantation in patients with occluded SFA occlusions. Five patients scheduled for BK amputations, successfully received overlapping infrapopliteal DES via this approach. After a mean follow-up period of 29 months, no deaths, no amputations, and no TLR were recorded. (Feiring and Wesolowski 2007).

The current generation DES used for BK interventions are balloon-expandable, which are known to be crushable. Stent crushing and fracturing may negatively influence stent performance and patient clinical outcome. To date, one case report of a fractured DES has been published (Scharzmaier-D'Assie et al 2007). The development of small vessel drug-eluting, self-expanding nitinol stents may improve the efficacy of the current generation of DES because self-expanding nitinol stents are less prone to fractures when compared with balloon-expandable stents. This can be important if longer infrapopliteal lesions will start to be treated. Our group recently reported the 12-month results using the commercially available non-drug-eluting Xpert $^{\circledR}$ (Abbott Vascular, Santa Clara, CA, USA) nitinol stent system in BK interventions. With a 12-month primary patency rate of $76.3 \%$, and a limb salvage rate of $95.9 \%$, the clinical outcome after bare metal nitinol stent implantation was remarkable. Moreover, 12-month angiography with quantative vessel analysis (QVA) on the $73 \%$ of patients available for follow-up in the cohort revealed an angiographic binary restenosis rate of only $20.5 \%$, which is comparable with well accepted coronary DES study outcomes. We attributed this optimal performance in the infrapopliteal arteries to the maintenance of flow dynamics because the stent was specifically designed for use in small vessels (Bosiers et al 2007). Although evidence currently indicates that the implantation of DES in the infrapopliteal vasculature leads to favorable outcomes with high mid-term primary patency and limb salvages rates, further support for the use of DES in patients with CLI and BK lesions will be gained from well-designed RCT. Such trials are about to start in conjunction with industry support.

\section{Renal arteries}

DES have also been applied for the treatment of renal artery stenosis, but as in the peripheral vascular bed, the number of publications remains sparse. Only 1 case report exists 
on the use of DES for bilateral renal stenting, Granillo and colleagues (2005) described a single case receiving a paclitaxel-eluting stent in both renal arteries. In another case report, Kakkar and colleagues (2006) reported on the implantation of a paclitaxel-eluting stent $\left(\operatorname{Scimed}^{\circledR}\right.$, Boston Scientific, Maple Grove, MN, USA) in a patient with recurrent renal artery in-stent restenosis. Angiographic control 6 months after index intervention showed continued wide patency of the treated vessel (Kakkar et al 2006).

In an editorial comment, Zeller and colleagues (2006) described the potential role of DES in the treatment of renal artery stenosis. They made reference to the GREAT trial (Palmaz Genesis peripheral stainless steel balloon expandable stent: comparing a sirolimus-coated vs.a bare stent in REnal Artery Treatment), the only trial which evaluates the efficacy of DES in renal artery disease. In this nonrandomized, nonblinded, prospective, multicenter observational study, the sirolimus-eluting Palmaz Genesis ${ }^{\mathrm{TM}}$ Peripheral Stent (Cordis, Miami, FL) is compared with its bare metal counterpart in 105 consecutive, patients with symptomatic renal artery disease. Sapoval and colleagues (2005) published beneficial results for the BMS arm of the study. Zähringer and colleagues (2007) published results from both arms of the study after 2 years of clinical follow-up. They reported that implantation of the sirolimus-eluting version led to an absolute reduction in the 6-month angiographic binary restenosis rate of $50 \%$ (BMS $=14.3 \%$, DES $=6.7 \%$; $\mathrm{p}=0.30)$ After 1 year, the target lesion revascularization rate was $11.5 \%$ in the BMS group compared with $1.9 \%$ in the drug-eluting group $(p=0.21)$. Implantation of both BMS and DES led to significant improvements in blood pressure, but $4 \%-7 \%$ patients had deterioration in renal function as measured by changes in serum creatinine. Despite, a trend toward improved outcome with DES in renal vasculature, the authors stated that the small sample size $(n=53)$ did not allow for detection of statistical significance between groups. Zeller and colleagues (2006) conclude that DES might be beneficial for patients with small renal arteries and impaired renal function. They believe that conventional BMS stent technology may lead to further deterioration in renal function because the need for reintervention exposes patients to recurrent administration of contrast and potential risk of renal embolism. According to their commentary, DES do not need to be used in patients with renal artery diameters greater or equal to $6 \mathrm{~mm}$ because results of existing endovascular interventions are already associated with a low restenosis rate in renal vasculature of this size. They state that use of DES may be preferable in patients with small diameter renal vessels ( $\leq 5 \mathrm{~mm}$ ), a single, functional kidney or bilateral renal artery stenosis with small vessels, where the tendency toward inferior outcomes may justify the added expense and the risk of intensified, prolonged antiplatelet therapy with acetylsalicylic acid and clopidogrel (Zeller et al 2006).

\section{Supra-aortic arteries Extracranial vasculature}

Gupta and colleagues (2006) reported outcomes of DES in the extracranial circulation. They retrospectively reviewed data from 59 patients with either extra- $(n=36)$ or intracranial stenoses $(n=29)$ treated with Cypher $^{\circledR}$ or Taxus ${ }^{\circledR}$ stents. The majority of the extracranial stents were implanted in the vertebral artery $(n=31)$ or the internal carotid artery (ICA) $(\mathrm{n}=5)$. Most $(95 \%)$ procedures were technically successful and the peri-procedural complication rate was 3\% (one nonflow limiting dissection and one stroke). Follow-up angiography $(n=41)$ or computed tomography $(n=7)$ performed at a median of 4 months post implantation revealed a restenosis rate of $6 \%$ (Gupta et al 2006). Although these results are early and retrospective, they provide hope for patients with cerebrovascular atherosclerosis who have a $19 \%$ risk of ipsilateral stroke when medically treated (Kasner et al 2006).

Two other authors have published case reports describing their experience with DES implanted for single extracranial lesions. Ko and colleagues (2004) mention in their report of 25 extracranial vertebral artery stenosis treated with balloonexpandable stents, the placement of one DES. At 1-month post-implantation, no adverse neurological events were observed (Ko et al 2004). In another case report NussbaumerOchsner and colleagues (2006) present a patient with preprocedural complaints of impaired left ocular perfusion and amaurosis fugax. The patient had occlusion at the origin of bilateral ICAs, stenoses of bilateral ECAs, and restenosis of the distal left CCA following prior endarterectomy. A Cypher $^{\circledR}$ DES was implanted in the left ECA followed by placement of a non-drug-eluting self-expanding nitinol stent (Precise, Cordis. Miami, FL, USA) in the left CCA. The procedure was uneventful, and the ocular symptoms resolved. At 6 months, the patient remained asymptomatic, with both stents being angiographically patent (Nussbaumer-Oschner et al 2006).

\section{Intracranial vasculature}

Abou-Chebl and colleagues (2005) collected data from 8 patients receiving a DES, 4 Cypher ${ }^{\circledR}$ and 4 Taxus ${ }^{\circledR}$ (Boston 
Scientific, Maple Grove, MN, USA). Stents were placed in the following locations: 3 intracranial ICA, 2 middle cerebral, 2 basilar, and 1 vertebral artery stenosis. Patients had a significant reduction in the initial stenosis (84\% to $2.5 \%)$. One patient had a retinal embolism during guide catheter removal and there was a post-stenting, nonflow-limiting, asymptomatic basilar artery dissection, which healed spontaneously during the follow-up period. No recurrence of cerebral ischemic events was observed during the follow-up period (mean 11.1 months). None of the angiographically studied patients ( $n=5$ of 8 ) developed significant restenosis, and none required target vessel revascularization (AbouChebl et al 2005).

In their retrospective analysis of patients treated with DES for both extra- and intracranial lesions, Gupta and colleagues (2006) described 29 intracranial cases treated with either Cypher ${ }^{\circledR}$ or Taxus ${ }^{\circledR}$ stents. In 19 cases, the lesions were in the intracranial vertebral or basilar artery, while the remaining 10 were in the intracranial ICA. Technically successful stent implantation was achieved in 90\% (26/29) of cases: the stent could not be deployed in 2 intracranial ICAs and 1 vertebral. Two peri-procedural complications were observed: 1 non-flow limiting dissection in an intracranial ICA and 1 disabling stroke 12 hours after placement of a basilar artery stent. There were no new restenotic lesions seen in 20 stents that were followed with repeat angiography or computed tomography at least 3 months after implantation (Gupta et al 2006).

Qureshi and colleagues (2006) retrospectively analysed 18 patients stented with DES for symptomatic lesions located in the intracranial ICA (6), the proximal middle cerebral artery (4), the vertebral artery (4), the vertebrobasilar junction (2), or the basilar artery (2). A Cypher ${ }^{\circledR}$ stent was used in 14 cases and the remaining 4 received Taxus ${ }^{\circledR}$ stents. It was attempted to perform stenting in 3 more patients, but the procedure was unsuccessful, resulting in technical success rate of $85.7 \%$. During the first month after the intervention, no patients died and 1 patient experienced a major stroke. Cerebral angiography was performed in 7 patients at 6 months follow-up and revealed a binary restenosis rate of 14\% (Qureshi et al 2006).

In our service, we performed a single Cypher ${ }^{\circledR}$ DES implantation in a 63-years old female patient presenting with a symptomatic high-grade stenosis situated in the intracranial segment of the ICA. Primary stenting was performed using a $3.5 \mathrm{~mm}$ diameter stent of $33 \mathrm{~mm}$ in length. The procedure was successfully completed without residual stenosis, or clinical complaints. To date, 2 years after the intervention, the patient is alive and has not experienced any further neurological events.

\section{Discussion}

At present, DES exist only on a balloon-mounted platform, which is expensive and potentially prone to crush injury. The expected superiority of DES for infrapopliteal and intracranial interventions in comparison to BMS is theoretical at present and extrapolated from outcomes seen in the coronary literature (Holmes et al 2004; Stone et al 2004). The need for performance of randomized controlled trials cannot be emphasized enough. The treatment of CLI, is one indication where use of DES may have a major impact. If treated strictly medically, only $50 \%$ of the patients with CLI will be alive without a major amputation after 1 year. Approximately $25 \%$ will have died and $25 \%$ will have had a major amputation (Norgren et al 2007; Siablis et al 2007). These patients lack conduit, have unsuitable target vessels for revascularization and significant comorbidities that preclude open surgical intervention. The paradigm has shifted to minimally invasive revascularization using PTA and stenting, but, although this approach has modified outcomes, the present $30 \%-50 \%$ restenosis rate at one year after PTA and implantation of BMS in the below-knee location warrants further improvement (Siablis et al 2005; Scheinert et al 2005; Commeau et al 2006).

There is also definitely room for improvement in the intracranial circulation. The compelling stroke rate seen in the WASID study, where patients with greater than $70 \%$ intracranial stenosis treated medically had a $23 \%$ stroke rate at one year, and, the $35 \%$ documented angiographic restenosis seen in the SSYLVIA trial, where the bare metal, balloonexpandable Neurolink ${ }^{\circledR}$ stent (Advanced Cardiovascular Inc., Guidant, Indianapolis, IN) was implanted, both highlight the limitations of existing treatment strategies (SSYLVIA Investigators 2004; Chimowitz et al 2005). Despite the high angiographic restenosis rate seen after BMS implantion in the SSYLVIA trial, only $40 \%$ of these restenosis led to neurologic complaints. It is therefore unclear, whether the potential for symptomatic restenosis reduction that might be provided with DES implants in this location outweighs the risk of early thombotic occlusion attributed to DES.

The initial enthusiasm for DES stems from their efficacy in reducing in-stent restenosis and neointimal hyperplasia from $30 \%$ to approximately 5\% (Holmes et al 2004; Stone et al 2004). Although direct arterial toxicity from the eluted drugs is unfounded, there are late thromboses secondary to delayed endothelialization that necessitate prolonged 
dual antiplatelet therapy and should temper nonchalant use of DES (Levy et al 2004; Moreno et al 2005). Unlike in animal models, use of DES in human atherosclerotic arteries results in delayed endothelialazation around the stent struts and thus makes the stent more susceptible to thrombosis. In coronary DES trials, the increased incidence of thrombosis with DES is small, in the order of $0.6 \%$ per annum, but, the accompanying 31\%-45\% mortality rate from the resultant myocardial infarction is of great concern (Harper 2004). Despite the fact that DES thrombosis in the peripheral circulation is not as lethal as in coronaries, dual antiplatelet therapy after DES is also warranted for PAOD to guarantee optimal stent performance and patient clinical well-being. Nevertheless, the use of dual antiplatelet therapy is related with an increased incidence (1\%-2\% per annum) of major bleeding complications and is expensive (Diener et al 2004; Eisenstein et al 2007). One year of clopidogrel costs approximately US\$1000.00. This expense is in addition to the cost already incurred by using a DES, which is usually fourfold greater than the bare metal equivalent; one coronary BMS costs US $\$ 800.00$ while one DES costs up to US\$2500.00 (Harper 2004). The duration of treatment with dual antiplatelet therapy after both coronary and peripheral implantation of DES remains a subject of ongoing debate. Based on emerging evidence, many cardiologists are recommending a minimum of 12 months of aspirin and clopidogrel, but, some are advocating indefinite use of dual antiplatelet therapy after DES implantation (Waksman 2006).

To overcome some of the shortcomings of the current generation of DES, further modifications in already existing stent technology need to be pursued, especially for infrapopliteal interventions. As CLI patients most often present with long, diffuse BK lesions, longer stents need to be manufactured to make use of DES more cost-effective in the BK vasculature. Especially for these longer lesions, there might be need for the development of specific infrapopliteal, drug-eluting, self-expanding nitinol stents to help circumvent potential crushing and strut fractures seen with the balloon-expandable platform (Scharzmaier-D'Assie et al 2007). One option involves development of drug-eluting bioabsorbable stents (Waksman 2006). The limitation of bioabsorbable stents has been the inflammatory response that is elicited after implantation. Modification of this response through local elution of anti-inflammatory, antiproliferative drugs should minimize restenosis, as well as inhibit injury-induced neointimal hyperplasia. As the requirement for vessel scaffolding provided by the stent is only needed temporarily, subsequent absorption of the stent should, in turn, allow endothelialazation, thus, precluding the need for ongoing antiplatelet therapy. (Waksman 2006) Another approach involves development of stents with inherent antithrombotic properties, such as stents coated with monoclonal antibodies capable of capturing circulating endothelial progenitor cells. (Harper 2004).

\section{Conclusion}

At present, the use of DES for noncoronary applications is investigational. Although there are good indications and some publications supporting the use of DES in several noncoronary vascular beds, more data and long-term followup are needed. The results from nonrandomized, controlled trials such as the ZILVER ${ }^{\circledR}$ PTX $^{\mathrm{TM}}$ and STRIDES trials are eagerly anticipated, but, ultimately, randomized controlled trials comparing DES with BMS implantation need to be performed to determine DES superiority. Until these results are available, review of the literature prompts us to make the following recommendations:

1. DES in the SFA provide no added benefit to the current generation of nitinol BMS, except, in complex lesions (long stenoses or occlusions). Additionally, in these type of complex lesions it will need to be investigated whether the potential improved patency and quality adjusted life year (QALY), outweigh the increased cost of (multiple) DES and the need for prolonged dual-antiplatelet therapy.

2. DES in the infrapopliteal vasculature of patients with CLI have the greatest potential benefit because the oneyear restenosis after implantation of BMS stents in this location is high (50\%) (Bosiers et al 2006). From a financial standpoint, there is existing coronary drug-eluting technology that can also be used in the infrapopliteal vasculature thus making this a more cost-effective undertaking. Preliminary results using DES in the infrapopliteal vasculature from single center studies are encouraging with low amputation rates $(<5 \%$ vs $>50 \%$ for untreated patients with CLI) (Siablis et al 2005; Bosiers et al 2006; Commeau et al 2006). There is need for RCTs and there are plans to perform these in conjuction with industry.

3. DES in renal interventions provides no added benefit to the current generation of balloon-expandable stents, except potentially in patients with solitary functional kidneys, where treatment failure has more compelling implications, or in patients with small renal arteries $(\leq 5 \mathrm{~mm})$ where outcomes are less favourable. Confirmation with RCTs is required.

4. Due to lack of evidence, DES in the intracranial and extracranial cerebrovascular vasculature is currently not supported. 


\section{Acknowledgments}

The authors take great pleasure in thanking the staff of Flanders Medical Research Program (www.fmrp.be), in particular Koen De Meester, who performed the systematic review of the literature and provided substantial support during the writing of the article. The authors report no conflicts of interest.

\section{References}

Abou-Chebl A, Bashir Q, Yadav JS. 2005. Drug-eluting stents for the treatment of intracranial atherosclerosis: initial experience and midterm angiographic follow-up. Stroke, 36:165-8.

Bosiers M, Deloose K, Verbist J, et al. 2006. Percutaneous transluminal angioplasty for treatment of "below-the-knee" critical limb ischemia: early outcomes following the use of sirolimus-eluting stents. J Cardiovasc Surg(Torino), 47:171-6.

Bosiers M, Deloose K, Verbist J, et al. 2007. Nitinol stenting for treatment of 'below-the-knee' critical limb ischemia: 1 year angiographic outcome of the Xpert stent. J Cardiovasc Surg(Torino), 48:455-61.

Chimowitz MI, Lynn MJ, Howlett-Smith H, et al. 2005. Warfarin-aspirin symptomatic intracranial disease trial investigators: comparison of warfarin and aspirin for symptomatic intracranial arterial stenosis. N Engl J Med, 352:1305-16.

Commeau P, Barragan P, Roquebert PO. 2006. Sirolimus for below the knee lesions: mid-term results of SiroBTK study. Catheter Cardiovasc Interv, 68:793-8.

Diener HC, Bogousslavsky J, Brass LM, et al.; MATCH Investigators. 2004. Aspirin and clopidogrel compared with clopidogrel alone after recent ischaemic stroke or transient ischaemic attack in high-risk patients (MATCH): randomised, double-blind, placebo-controlled trial. Lancet, 364:331-7.

Duda SH, Pusich B, Richter G, et al. 2002. Sirolimus-eluting stents for the treatment of obstructive superficial femoral artery disease: Six-month results. Circulation, 106:1505-9.

Duda SH, Bosiers M, Lammer J, et al. Sirolimus-eluting versus bare nitinol stent for obstructive superficial femoral artery disease: The SIROCCO II trial. J Vasc Interv Radiol, 16:331-8.

Duda SH, Bosiers M, Lammer J, et al. 2006. Drug-eluting and bare nitinol stents for the treatment of atherosclerotic lesions in the superficial femoral artery: Long-term results from the SIROCCO trial. J Endovasc Ther, 13:701-10.

Eisenstein EL, Anstrom KJ, Kong DF, et al. 2007. Clopidogrel use and long-term clinical outcomes after drug-eluting stent implantation. JAMA, 297:159-68.

Feiring AJ, Wesolowski AA, Lade S. 2004. Primary stent-supported angioplasty for treatment of below-knee critical limb ischemia and severe claudication: early and one-year outcomes. J Am Coll Cardiol, 44:2307-14.

Feiring AJ, Wesolowski AA. 2007. Antegrade popliteal artery approach for the treatment of critical limb ischemia in patients with occluded superficial femoral arteries. Catheter Cardiovasc Intervi, 69:665-70.

Granillo GA, van Dijk LC, McFadden EP, et al. 2005. Percutaneous radial intervention for complex bilateral renal artery stenosis using paclitaxel eluting stents. Catheter Cardiovasc Interv, 64:23-7.

Gupta R, Al-Ali F, Thomas AJ, et al. 2006. Safety, feasibility, and shortterm follow-up of drug-eluting stent placement in the intracranial and extracranial circulation. Stroke, 37:2562-6.

Harper RW. 2007. Drug-eluting stents coronary stents - a note of caution. Med J Aust, 186:253-5.

Hill R, Bagust A, Bakhai A, et al. 2004. Coronary artery stents: a rapid systematic review and economic evaluation. Health Technol Assess, 8(35):iii-iv, 1-242.
Holmes DR, Leon MB, Moses JW, et al. 2004. Analysis of 1-year clinical outcomes in the SIRIUS trial: a randomized trial of a sirolimus-eluting stent versus a standard stent in patients at high risk for coronary restenosis. Circulation, 109:634-40

Kakkar AK, Fischi M, Narins CR. 2006. Drug-eluting stent implantation for treatment of recurrent renal artery in-stent restenosis. Catheter Cardiovasc Interv, 68:118-22; discussion 123-4.

Kasner SE, Chimowitz MI, Lynn MJ, et al. 2006. Warfarin Aspirin symptomatic intracranial disease trial. Circulation, 113:555-63.

Ko Y-G, Park S, Kim J-Y, et al. 2004. Percutaneous interventional treatment of extracranial vertebral artery stenosis with coronary stents. Yonsei Med J, 45:629-34.

Levy EI, Hanel RA, Howington JU, et al. 2004. Sirolimus-eluting stents in the canine cerebral vasculature: a prospective, randomized, blinded assessment of safety and vessel response. J Neurosurg, 100:688-94.

Mauri L, Orav EJ, Candia SC, et al. 2005. Robustness of late lumen loss in discriminating drug-eluting stents across variable observational and randomized trials. Circulation, 112:2833-9.

Moreno R, Fernandez C, Hernandez R, et al. 2005. Drug-eluting stent thrombosis: results from a pooled analysis including 10 randomized studies. J Am Coll Cardiol, 45:954-9.

Norgren L, Hiatt WR, Dormandy JA, et al. 2007. TASC II working group. Intersociety consensus for the mangement of peripheral arterial disease (TASC II). Eur J Vasc Endovasc Surg, 33(Suppl 1):S1-75.

Nussbaumer-Ochsner Y, Eberli FR, Baumgartner RW, et al. 2006. Sirolimus-eluting stenting of the external carotid artery for the treatment of ocular ischemia. J Endovasc Ther, 13:672-5.

Qureshi AI, Kirmani JF, Hussein HM, et al. 2006. Early and intermediate-term outcomes with drug-eluting stents in high-risk patients with symptomatic intracranial stenosis. Neurosurgery, 59:1044-51.

Roiron C, Sanchez P, Bouzamondo A, et al. 2006. Drug eluting stents: an updated meta-analysis of randomised controlled trials. Heart, 92:641-9.

Sapoval M, Zahringer M, Pattynama P, et al. 2005. Low-profile stent system for treatment of atherosclerotic renal artery stenosis: The GREAT trial. J Vasc Interv Radiol, 16:1195-202.

Scheinert D, Ulrich M, Scheinert S, et al. 2006. Comparison of sirolimuseluting vs. bare-metal stents for the treatment of infrapopliteal obstructions. EuroInterv, 2:169-74.

Schwarzmaier-D'Assie A, Karnik R, et al. 2007. Fracture of a drugeluting stent in the tibioperoneal trunk following bifurcation stenting. J Endovasc Ther, 14:106-9.

Siablis D, Kraniotis P, Karnabatidis D, et al. 2005. Sirolimus-eluting versus bare stents for bailout after suboptimal infrapopliteal angioplasty for critical limb ischemia: 6-month angiographic results from a nonrandomized prospective single-center study. J Endovasc Ther, 12:685-95.

Siablis D, Karnabatidis D, Katsanos K, et al. 2007. Sirolimus-eluting versus bare stents after suboptimal infrapopliteal angioplasty for critical limb ischemia: enduring 1-year angiographic and clinical benefit. J Endovasc Ther, 14:241-50.

Stone GW, Ellis SG, Cox DA, et al. 2004. One-year clinical results with the slow-release, polymer-based, paclitaxel-eluting TAXUS stent: the TAXUS-IV trial. Circulation, 109:1942-7.

SSYLVIA Investigators. 2004. Stenting of symptomatic atherosclerotic lesions in the vertebral or intracranial arteries (SSYLVIA): study results. Stroke, 35:1388-92.

Waksman R. 2006. Update on bioabsorbable stents: From bench to clinical. J Interven Cardiol, 19:414-21.

Zähringer M, Sapoval M, Pattynama PMT, et al. 2007. Sirolimus-elutinG versus bare-metal low-profile stent for REnal Artery Treatment (GREAT trial): Angiographic follow-up after 6 month and clinical outcome up to 2 years. J Endovasc Ther, 14:460-8.

Zeller T, Rastan A, Rothenpieler U, et al. 2006. Restenosis after stenting of atherosclerotic renal artery stenosis: Is there a rationale for the use of drug-eluting stents? Catheter Cardiovasc Interv, 68:125-30. 
